\title{
Paediatric radial head dislocation with associated ulnar pathology
}

\begin{abstract}
Radial head dislocations are uncommon injuries. Most reported cases in the literature involve missed/chronic injuries, congenital dislocations or cases with associated ulnar bowing/fractures. A high index of clinical suspicion and subsequent urgent management is of upmost importance to avoid a missed diagnosis and subsequent morbidity, namely significant elbow pain, deformity, stiffness and nerve palsies.
\end{abstract}

Keywords: Radial head, Dislocation, Plastic deformation, Radial neck
Volume 8 Issue 2 - 2017

\author{
Cary Fletcher \\ Saint Ann's Bay Regional Hospital, Jamaica
}

Correspondence: Cary Fletcher, Saint Ann's Bay Regional Hospital, Saint Ann's Bay, Jamica, Tel 876-972-2273,

Email c.fletch30@yahoo.com

Received: February 13, 2017| Published: May 01, 2017

\section{Introduction}

Radial head dislocations with associated ulna pathology are rare injuries, but they are far more common than isolated radial head dislocations. ${ }^{1,2}$ Although the vast majority of acute paediatric upper extremity fractures and dislocations are successfully treated by closed means, certain injuries will require operative management. Failed attempts of closed management of paediatric injuries are often an indication for surgery. ${ }^{3}$

\section{Discussion}

For all age groups, anterior dislocation of the radial head may either be seen in combination with an ulnar fracture (Monteggia fracture) or without any fracture (Monteggia equivalent). ${ }^{4}$ In children, it is felt that many radial head dislocations are associated with ulnar bowing. Some authors advocate that this bowing deformity must be surgically addressed in order to achieve a reduction. ${ }^{1}$ Hudson et al. ${ }^{5}$ stated that Monteggia fractures are more common than isolated radial head dislocations as well as injuries involving associated ulnar bowing. The ulnar bowing is facilitated by the plasticity of the ulna in children hence the bone may undergo significant bending without fracturing. ${ }^{5}$ Plastic bony resilience distinguishes children from adults, hence why the adult will more likely suffer an associated displaced ulna fracture. ${ }^{2}$ The usual mechanism of injury is a fall on the outstretched hand with the elbow fully extended and the forearm pronated with a strain applied to the elbow. ${ }^{1,6}$ These injuries are rare in children and are easily missed due to the history often being vague. ${ }^{4}$

Plastic deformation should be suspected in children presenting with deformity but has no radiological evidence of a fracture. ${ }^{2}$ Also, the radiocapitellar line (to assess for radiocapitellar dislocation) and the ulnar bow sign should routinely be radiologically assessed. Reports of isolated radial head dislocations in the literature have been intermittent and consisting of case reports or small case series whereby impressions have often been inconclusive. ${ }^{7}$ Diagnosis is best made radiographically with a true lateral X-Ray showing the elbow and wrist joints. ${ }^{2}$

The annular ligament has been noted to be the most important structure in maintaining the stability of the radial head. Once radial head dislocation occurs, this ligament must be completely disrupted. The interosseous membrane, quadrate ligament, and the oblique cord are other supporting structures playing a lesser role. The interosseous membrane, anterior fibres of the quadrate ligament and oblique cord are all taut in supination. The posterior fibres of the quadrate ligament are taut in pronation. ${ }^{5}$

A persistently dislocated radial head may result in an increasing valgus deformity of the elbow and subsequent radial and ulnar nerve abnormalities. Obstruction and malformation of the radial head often results in decreased elbow flexion and loss of forearm rotation. ${ }^{8,9}$ In some cases, the children are initially able to adapt to the forearm disability and have minimal symptoms however; the disability and the deformity become more apparent with skeletal growth. ${ }^{7}$

The vast majority of acute injuries are successfully managed via closed reduction. ${ }^{10}$ In his case series, Hudson et al. ${ }^{5}$ successfully managed all patients who presented with acute isolated radial head dislocations by closed means. Both patients who required open reduction in his series presented six months post injury.

For surgical management, Bucknill et al. ${ }^{7}$ has one of the largest series in the literature, whereby he had good outcomes in twenty eight patients. All presented three months or later and the average patient age was 7 years old. Seventeen patients presented with deformity and all twenty eight patients presented with decreased range of motion namely flexion and pronation. Eight underwent annular ligament reconstruction using the triceps tendon and the reduction was held with a Kirshner wire. He noted that ulnar osteotomy was required in some cases to facilitate reduction. He also recommended against radial ostoeotomy with the rationale that adhesions to the radius will limit forearm rotation. While numerous authors have had good results with annular ligament reconstruction, the general principle in treating this injury is that the surgeon keep procedures to a minimum and performs the least invasive procedure in order to achieve a stable, congruent reduction. ${ }^{6}$

\section{Conclusion}

It is of utmost importance to diagnose radial head dislocations with or without associated ulnar pathology as early as possible to avoid possible sequalae of persistent deformities, nerve palsies and impaired upper limb function. Treatment should be as minimally invasive as possible because most are successfully treated by closed means. Surgery is indicated only when closed management fails.

\section{Acknowledgements}

Thanks to Kaye Patrice Lambert for her valuable technical support. 


\section{Conflicts of interest}

None.

\section{References}

1. Bradley MP, Tashjian RZ, Eberson CP. Irreducable radial head dislocation in a child: a case report. Am J Orthop. 200736(5):E76-E79.

2. Eygendaal D, Hillen RJ. Open reduction and corrective ulnar osteotomy for missed radial head dislocations in children. Strat Traum Limb Recon. 20072(1):31-34.

3. Chen WS. Late neuropathy in chronic dislocation of the radial head. Report of two cases. Acta Orthop Scand. 1992;63(3):343-344.

4. Dormans JP, Rang M. The problem of Monteggia fracture-dislocations in children. Orthop Clin North Am. 1990;21(2):251-266.

5. Hudson DA, De Beer. Isolated traumatic dislocation of the radial head in children. J Bone and Joint Surg. 1986;68-B(3):378-381.
6. Bucknill TM. Anterior dislocation of the radial head in children. Roy Soc Med. 1977;70(9):620-624.

7. Goyal S, Hussain A. Anterior dislocation of the radial head with plastic bowing of the ulna introducing modification of Bado's classification. JK-practitioner. 2005;12(1):20-23.

8. Gupta V, Kundu ZS, Sangwan SS, et al. Isolated post-traumatic radial head dislocation, a rare and easily missedinjury-a case report. Malaysian Orthopaedic Journal. 2013;1(1):74-78.

9. Keminitz S, Schrijver FD, Smet DE. Radial head dislocation with plastic deformation of the ulna in children. Acta Orthopaedica Belgica. 2000;66(4):359-362.

10. Belangero WD, Livani B, Zogaib RK. Treatment of chronic radial head dislocations. International Orthopaedics. 2007;31(2):151-154. 